\title{
Characterisation of a Computationally Defined Treatment Target for Anxiety and Depression
}

\author{
Michael Browning and Erdem Pulcu, University of Oxford
}

\section{Background}

Preferential learning from negative at the expense of positive events, has been causally linked to anxiety and depression. This suggests that interventions which target such negative learning bias may reduce symptoms of the illness, although the best way to achieve this is not clear. Recent computational work suggests that people preferentially learn from outcomes with high information content (i.e. which improve prediction of the future), and that central norepinephrine acts to report the information content of the outcomes. We tested whether it was possible to manipulate learning bias and associated central norepinephric activity by controlling the information content of positive and negative events in a computer based task.

\section{Methods}

In the study 30 non-clinical participants completed a learning task in which the information content of positive and negative outcomes (wins and losses) was independently manipulated by controlling their volatility. Pupilometry data was collected to assess

central norepinephrine activity. A simple computational model was used to estimate learning rate for wins and losses.

\section{Results}

Participants demonstrated a significant increase in learning rate to the particular event (wins or losses) which carried more information $(\mathrm{F}=52, \mathrm{p}<0.01)$. Activity of the central norephipheric system was similarly modified by the intervention $(\mathrm{p}<0.01)$.

\section{Conclusions}

These results indicate that people maintain separate estimates of the information content of positive and negative outcomes, and these estimates may be targeted by a simple cognitive intervention. In an ongoing randomised clinical study the effects of this intervention on symptoms of depression is being tested. 